\title{
A Multi-Orientation Error Separation Technique for Spindle Metrology of Miniature Ultra-High-Speed Spindles
}

\author{
K. Prashanth Anandan ${ }^{\mathrm{a}, *}$, O. Burak Ozdoganlar ${ }^{\mathrm{a}, * *}$ \\ ${ }^{a}$ Department of Mechanical Engineering, Carnegie Mellon University, Pittsburgh, \\ Pennsylvania 15213, USA
}

\begin{abstract}
This paper presents a multi-orientation error separation technique to remove the artifact form error from the radial measurements to obtain the radial spindle error motions of miniature ultra-high-speed (UHS) spindles. Unlike the existing approaches, the present technique neither relies on high-accuracy fixtures, nor necessitates measurements from specific orientations of the artifact. Rather, the spindle error motions are measured from a set of arbitrary artifact orientations using laser Doppler vibrometry (LDV). The angle of each artifact-setup orientation with respect to the spindle is determined with high precision through reflectivity measurement of the marks made on both the artifact and the spindle using another LDV. Although the presented approach can be applied by using different sensors (e.g., capacitance probes), we demonstrate the approach using LDVs. With the displacement measurement direction fixed, measurements are conducted from both LDVs for multiple orientations of the artifact. Using the unique implementation scheme developed in this paper, data from these orientations are post-processed to compute the artifact form error and further remove it from the radial motion measurements to obtain the synchronous radial spindle error motions. A thorough experimental evaluation is presented to
\end{abstract}

\footnotetext{
${ }^{*}$ Currently at Bruker Nano Surfaces, Santa Barbara, CA

** Corresponding author:

Email address: ozdoganlar@cmu.edu (O. Burak Ozdoganlar)

${ }^{1}$ Phone:1-412-268-9890

${ }^{2}$ Fax:1-412-268-3348
}

Preprint submitted to Elsevier

June 24, 2015

(C) 2015. This manuscript version is made available under the Elsevier user license http://www.elsevier.com/open-access/userlicense/1.0/ 
quantify both the repeatability of the measured artifact form errors as well as the bandwidth of error separation for various number of artifact orientations. The spindle error motions measured from both the sphere and stem portions of a custom fabricated sphere-on-stem artifact mounted on a typical miniature UHS spindle, are seen to be similar in shape and within $5 \mathrm{~nm}$ in magnitude across the revolution, thus demonstrating the effectiveness of the technique. Using this technique, spindle error motions at ultra-high speeds up to $150 \mathrm{krpm}$ were successfully quantified. Although the implementation scheme is demonstrated for miniature UHS spindles, it is readily applicable for error separation on macro-scale spindles without the need for any high-precision fixtures and precise setting of angles.

Keywords: Ultra-high-speed spindles, spindle metrology, error separation, multi-orientation method, micro-manufacturing, micromachining.

\section{Introduction}

Among many other factors, dimensional and surface accuracy of precision machining operations depend on the accuracy of the rotary motions provided by the spindle. Miniature ultra-high-speed (UHS) spindles are widely used for micromachining and precision machining applications, and the strict tolerance and accuracy requirements of those processes impose limits on the unwanted axial and radial motions encountered when using the UHS spindles.

In spindle metrology, the motions of the spindle are measured from a precision artifact attached to the spindle. In addition to the synchronous and asynchronous spindle error motions, the measured radial motions also include the surface profile (form error or out-of-roundness) of the artifact around the circumferential measurement track. In order to isolate the spindle error motions from the form error of the artifact, and thus, to accurately determine the spindle error motions, error separation techniques are used. Various techniques that have been developed for error separation can be classified into (1) reversal techniques [4-9], (2) multi-probe techniques [8-12], and (3) multi-step techniques 
$[9,13-15]$. A thorough description of these techniques is provided in $[4,6]$. Common to all of the error separation techniques presented in the literature is the requirement of precise and rigid fixturing, with accurate and precise angular positioning of displacement sensors. Under ideal conditions, including perfect alignment, positioning, and fixturing, the reversal techniques allow for perfect separation of artifact and spindle errors. However, this is only true in theory since such ideal conditions cannot be obtained in reality. Even under ideal conditions, due to the finite number of indexing probes/angles, the multi-probe and multi-step techniques cannot completely separate certain harmonics of the spindle and artifact errors. This issue is commonly referred to as harmonic suppression $[6,14,17]$.

Many works in the literature have attempted to address the harmonic suppression problem. Gao et al. [16] showed that a method based on measuring the radial slope (using an angle probe) simultaneously with radial displacements could minimize the harmonic suppression problem and improve the accuracy of error motion measurement. Many researchers [6, 8, 13, 15, 25] have also shown that, while full separation of the artifact form and spindle error motion cannot be obtained using the multi-probe and multi-step techniques, sufficient number of harmonics can be effectively separated by a judicious choice of the angles and the number of probes/steps. Although these techniques have been successfully implemented to measure spindle error motions of ultra-precision spindles at sub-nanometer levels [8, 9, 25], their application imposes significant practical challenges during implementation due to the need for sophisticated measurement setups (as shown in [16]) or extremely precise fixturing to prescribe the chosen angles.

Application of the well-established spindle metrology techniques to miniature UHS spindles poses important challenges arising from (1) the smaller size of the spindle and the artifact (typical artifact size is $\phi 3 \mathrm{~mm}$ or $\phi 0.125 \mathrm{in}$.); (2) the curvature effect due to the small artifact diameter; and (3) the requirement for performing measurements at higher spindle speeds (e.g., $>40 \mathrm{krpm}$ ), since majority of the UHS spindles cannot be operated below a specific speed. Fur- 
thermore, the sphere-on-stem artifacts typically used for these measurements in the macro-scale are not commercially available for the $\phi 3 \mathrm{~mm}$ size and are very expensive to fabricate. To address those challenges, the authors recently developed a laser Doppler vibrometer (LDV)-based measurement approach for measuring axial and radial spindle motions of ultra-high-speed spindles [1, 2]. In those works, however, the form error of the artifact was not removed, but rather considered as a source of uncertainty in the synchronous component of the radial error, thus leading to relatively high uncertainties in the measurement of radial synchronous spindle error motions. This approach was followed since, due to the aforementioned challenges, error separation techniques presented in the literature were either impossible or impractical to apply to UHS spindles. Therefore, an effective error separation technique that will enable accurate measurement of error motions of miniature UHS spindles at operational speeds is critically needed. Ideally, this technique will alleviate the need for custom designed high precision artifacts, and will capture many harmonics (e.g., > 50) to enable high precision measurement of the synchronous spindle error motions of miniature UHS spindles.

In this work, we have implemented a single-probe multi-orientation technique to remove the artifact form error from the radial measurements to obtain the radial spindle errors of miniature UHS spindles. The approach involves measurements of radial motions using a single LDV by keeping it stationary, and conducting measurements at multiple orientations of the artifact (i.e., by removing the artifact, changing its relative angular position with respect to the spindle, and re-attaching the artifact in its re-oriented configuration). While the idea of multi-orientation error separation (MOES) is not new [9, 13-15], we have established an unique implementation approach that eliminates the need for measuring at specific orientations, but rather uses measurements from multiple arbitrary orientations of the artifact, thereby circumventing the need for extra precision fixtures. This is enabled by accurate determination of the relative orientation between the artifact and the spindle by monitoring the reflectivity signal of another laser in-situ. We demonstrate the use of MOES technique for 
the measurement of the spindle error motions of a typical miniature UHS spindle across its operational speeds. Furthermore, the effectiveness of the technique is evaluated by comparing spindle error motions determined from measurements from two artifact surfaces with drastically different form errors. It is noted here that, although the implementation scheme has been demonstrated for miniature UHS spindles, the same approach can be readily used for error separation on macro-scale and conventional spindles.

This paper is organized as follows: First, a brief introduction of the MOES technique is provided. Then, the details of the implementation scheme developed in this work are described, with a focus on measuring spindle error motions of UHS spindles. Next, using a typical UHS spindle, measurements are conducted to (a) demonstrate the functionality of the implementation scheme by measuring spindle error motions from the sphere and stem portions of the sphere-on-stem artifact, and (b) study the effect of the number of orientations (used for error separation) on both the repeatability and bandwidth of error separation. Finally, to demonstrate the effectiveness of the implementation scheme across a wide range of speeds, radial spindle error motions of the tested UHS spindle are obtained at four speeds $(40 \mathrm{krpm}, 90 \mathrm{krpm}, 120 \mathrm{krpm}$, and 150 $\mathrm{krpm}$ ) that span the entire operating range of the spindle along both the fixedand rotating-sensitive directions.

\section{Description of the Single-Probe Multi-Orientation Technique}

The theory for single-probe multi-orientation technique was first described in [6]. This technique requires measurements from at least two orientations of the artifact. It involves using a single displacement sensor to measure the radial motions from the surface of an artifact that is attached to an axis of rotation provided by the spindle. The motions measured when the spindle is run at the operating speeds can be decomposed into motions at the fundamental frequency, synchronous motions and asynchronous motions [19, 20]. The synchronous motions consist of the artifact form error and the speed-dependent synchronous 

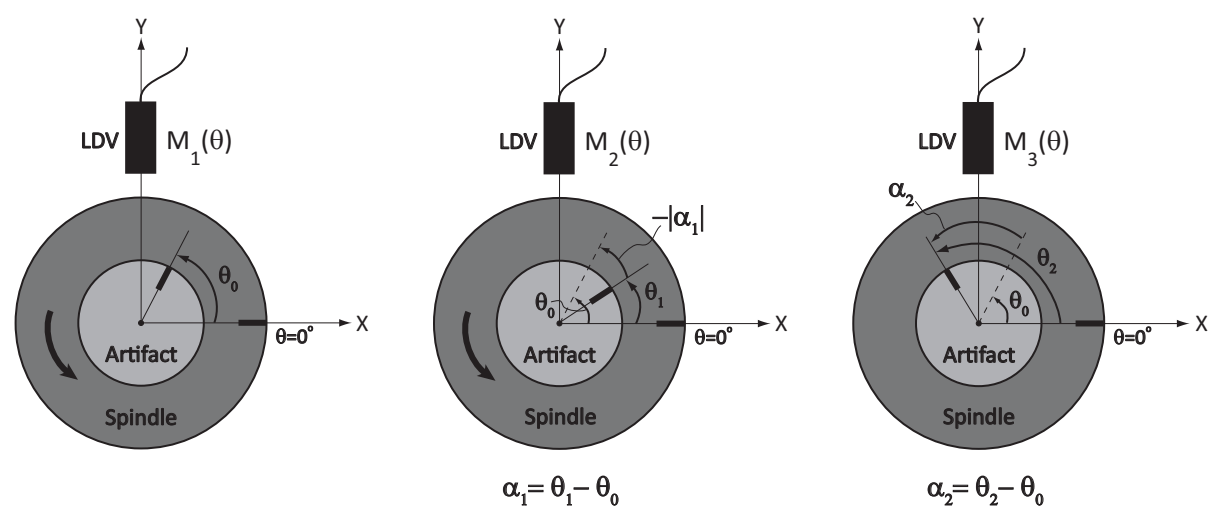

Figure 1: Steps involved in Multi-Orientation Technique when using three re-orientations.

spindle error motions $[19,20]$. In order to obtain the true synchronous spindle error motions, the synchronous radial motion measurements from multiple orientations of the artifact are combined to calculate and separate the artifact form error.

A description of the steps involved in the multi-orientation technique is shown in Fig. 1 for an implementation along the fixed-sensitive $Y$-direction. After conducting the first measurement, the artifact is re-oriented by removing it from the spindle, rotating by a certain angle about its axis, and re-attaching it to the spindle for the second measurement. This step is repeated until all the measurements from the desired number of orientations are completed. The measured displacement data from each orientation is post-processed to obtain the synchronous radial motions at certain fixed angular positions of the spindle.

Denoting the spindle error motions and artifact form error measured along the fixed-sensitive $Y$-direction as $S(\theta)$ and $A(\theta)$, respectively, the synchronous radial motions for the three orientations $M_{1}(\theta), M_{2}(\theta), M_{3}(\theta)$ can be related to $S(\theta)$ and $A(\theta)$ as

$$
\begin{aligned}
& M_{1}(\theta)=A(\theta)+S(\theta) \\
& M_{2}(\theta)=A\left(\theta-\alpha_{1}\right)+S(\theta) \\
& M_{3}(\theta)=A\left(\theta-\alpha_{2}\right)+S(\theta),
\end{aligned}
$$


where $\alpha_{1}$ and $\alpha_{2}$ are the rotation angles of the artifact (with respect to the first orientation) in the second and third orientations, respectively, as shown in Fig. 1. It is important to note that the angular reference $(\theta=0)$ between different orientations corresponds to the same physical angular orientation of the spindle.

Mathematically, we can eliminate the spindle error motions $S(\theta)$ from the three measurements and obtain a relationship for the artifact form error as follows: Consider a combined sum $T(\theta)$ which can be written as

$$
\begin{aligned}
T(\theta) & =2 M_{1}(\theta)-M_{2}(\theta)-M_{3}(\theta) \\
& =2 A(\theta)-A\left(\theta-\alpha_{1}\right)-A\left(\theta-\alpha_{2}\right) .
\end{aligned}
$$

Each term in the above equation is a continuous function; during the measurement, the values of each function is obtained at discrete points as dictated by the sampling rate of the digital data acquisition. However, it is also recognized that each function is periodic by definition. As such, a frequency domain analysis can be considered by applying Fourier series expansion of the respective terms as

$$
\mathcal{F}[T(\theta)]=2 \mathcal{F}[A(\theta)]-\mathcal{F}\left[A\left(\theta-\alpha_{1}\right)\right]-\mathcal{F}\left[A\left(\theta-\alpha_{2}\right)\right],
$$

where $\mathcal{F}[*]$ represents the continuous Fourier series of the term in the parenthesis. Since $A(\theta)$ is periodic with a period $2 \pi$, the continuous Fourier series of $A(\theta)$ can be expressed as

$$
\mathcal{F}[A(\theta)]=\sum_{k=-\infty}^{\infty} A_{k} e^{j k \theta}
$$

where $A_{k}$ are the Fourier series coefficients given by

$$
A_{k}=\frac{1}{2 \pi} \int_{0}^{2 \pi} A(\theta) e^{-j k \theta} d \theta
$$


Further,

$$
\mathcal{F}[A(\theta-\alpha)]=\sum_{k=-\infty}^{\infty} e^{-j k \alpha} A_{k} e^{j k \theta}
$$

Using this, Eq.(3) can be written as

$$
\sum_{k=-\infty}^{\infty} T_{k} e^{j k \theta}=\sum_{k=-\infty}^{\infty}\left[2-e^{-j k \alpha_{1}}-e^{-j k \alpha_{2}}\right] A_{k} e^{j k \theta}
$$

This expression can be written in terms of the Fourier series coefficients $T_{k}$ and $A_{k}$ of $T(\theta)$ and $A(\theta)$, respectively, as

$$
T_{k}=A_{k}\left[2-e^{-j k \alpha_{1}}-e^{-j k \alpha_{2}}\right] .
$$

Therefore, $A_{k}$ can be obtained as

$$
A_{k}=\frac{T_{k}}{\left[2-e^{-j k \alpha_{1}}-e^{-j k \alpha_{2}}\right]} .
$$

Since synchronous motion data is obtained at a finite number of angular locations $\left(\theta_{i}\right.$, with $i=1,2, \ldots N$, where $N$ is the total number of angular sampling points per revolution), the artifact form error $A\left(\theta_{i}\right)$ at these discrete $\theta_{i}$ s can be expressed by taking the inverse discrete Fourier transform of $A_{k}$ as

$$
A\left(\theta_{i}\right)=\sum_{k=1}^{N} A_{k} \exp \left(j \frac{2 \pi}{N} k i\right)
$$

The spindle error motion at $\theta_{i}$ can then be calculated as

$$
S\left(\theta_{i}\right)=M_{1}\left(\theta_{i}\right)-A\left(\theta_{i}\right) .
$$

It can be seen from Eq.(9) that the denominator approaches to zero for certain values of $k$ that satisfy $k \alpha_{1}=2 \pi N_{1}$ and $k \alpha_{2}=2 \pi N_{2}$ simultaneously, where $N_{1}$ and $N_{2}$ are integers. The artifact form error, and hence the spindle 
error motion, cannot be separated at those $k^{\text {th }}$ harmonics. This is the main drawback of the multi-orientation technique. The harmonics that cannot be separated are commonly referred to in the literature as the harmonic losses, and this issue is referred to as harmonic suppression. The highest value of $k$ until which all the harmonics are separated can be referred to as the bandwidth of the error separation technique. One way to alleviate this issue, for a given number of orientations, is to choose specific orientation angles (or range of angles) [8, 17]. Alternatively, the bandwidth can be increased by increasing the number of orientations, as suggested in [6]. For example, if $m$ orientations are used, the equation for calculating the artifact form error becomes

$$
A_{k}=\frac{T_{k}}{(m-1)-\sum_{c=1}^{m-1} e^{-j k \alpha_{c}}}
$$

where $T_{k}$ s are the Fourier coefficients of the combined sum, $T(\theta)$ calculated as

$$
T(\theta)=(m-1) M_{1}(\theta)-\sum_{c=1}^{m-1} M_{c+1}(\theta)
$$

and $\alpha_{c}$ is rotation angle of the artifact in the $(c+1)^{t h}$ orientation with respect to the first orientation. For an arbitrary set of rotation angles, the first value of $k$ at which $\sum_{c=1}^{m-1} e^{-j k \alpha_{c}}=(m-1)$ is higher for a larger $m$, thus enabling obtaining a higher bandwidth with greater number of orientations.

For practical purposes, instead of letting the denominator become zero (and thus, each exponential $e^{-j k \alpha_{c}}$ become equal to 1 simultaneously), it is more practical (and conservative) to define the bandwidth as the lowest value of $k$ for which each exponential $e^{-j k \alpha_{c}}$ approaches a value $\Delta$ that is close to 1 simultaneously. In this paper, we will adopt this approach and set $\Delta=0.85$ for determining the bandwidth. 


\section{Implementation of the multi-orientation technique}

\subsection{Measurement setup}

The measurement setup used for implementation of the multi-orientation technique on UHS spindles is shown in Fig. 2 (see [1, 2] for further details of the overall setup). A sphere-on-stem artifact is attached to the miniature UHS spindle being tested. The artifact uses a Grade 3 hardened steel sphere (with a specified sphericity of $76 \mathrm{~nm}$ ) attached to a stem (a Class-XX steel gage pin) with a specified cylindricity of $254 \mathrm{~nm}$. Prior to testing, physical marks are created (by engraving and coating with black paint) at arbitrary angular positions on the stem portion of the artifact and on a rotating surface integral to the spindle. Each marks is roughly $1 \mathrm{~mm}$ wide and $100 \mu \mathrm{m}$ deep.

Two LDV systems are used for the implementation of the multi-orientation technique. The laser beam spot size of each LDV is approximately $30-50 \mu \mathrm{m}$. For the particular decoder (Polytec DD-500) used, the LDVs can measure displacements at picometer-level resolution with a frequency bandwidth up to 350 $\mathrm{kHz}$. One of the LDV systems is used in the single-point measurement mode to measure the radial displacement along the desired fixed-sensitive direction (e.g., the Y-direction shown in Fig. 2) from the surface of the sphere portion of the sphere-on-stem artifact.

The second LDV system is used in the relative-mode to measure the relative reflectivity ${ }^{3}$ between the stem surface of the sphere-on-stem artifact and the rotating surface integral to the spindle. When the LDV is used in this mode, a single laser source is split into two laser beams, each of which is focused independently on the different measurement surfaces. The relative reflectivity signal is post-processed (as explained in the next section) to obtain the relative angle between the artifact and spindle for a given artifact attachment, which indicates the current orientation of the artifact.

\footnotetext{
${ }^{3}$ In addition to measuring displacements, the LDV controller also outputs a voltage signal proportional to the intensity of the reflected signal from the measurement location.
} 


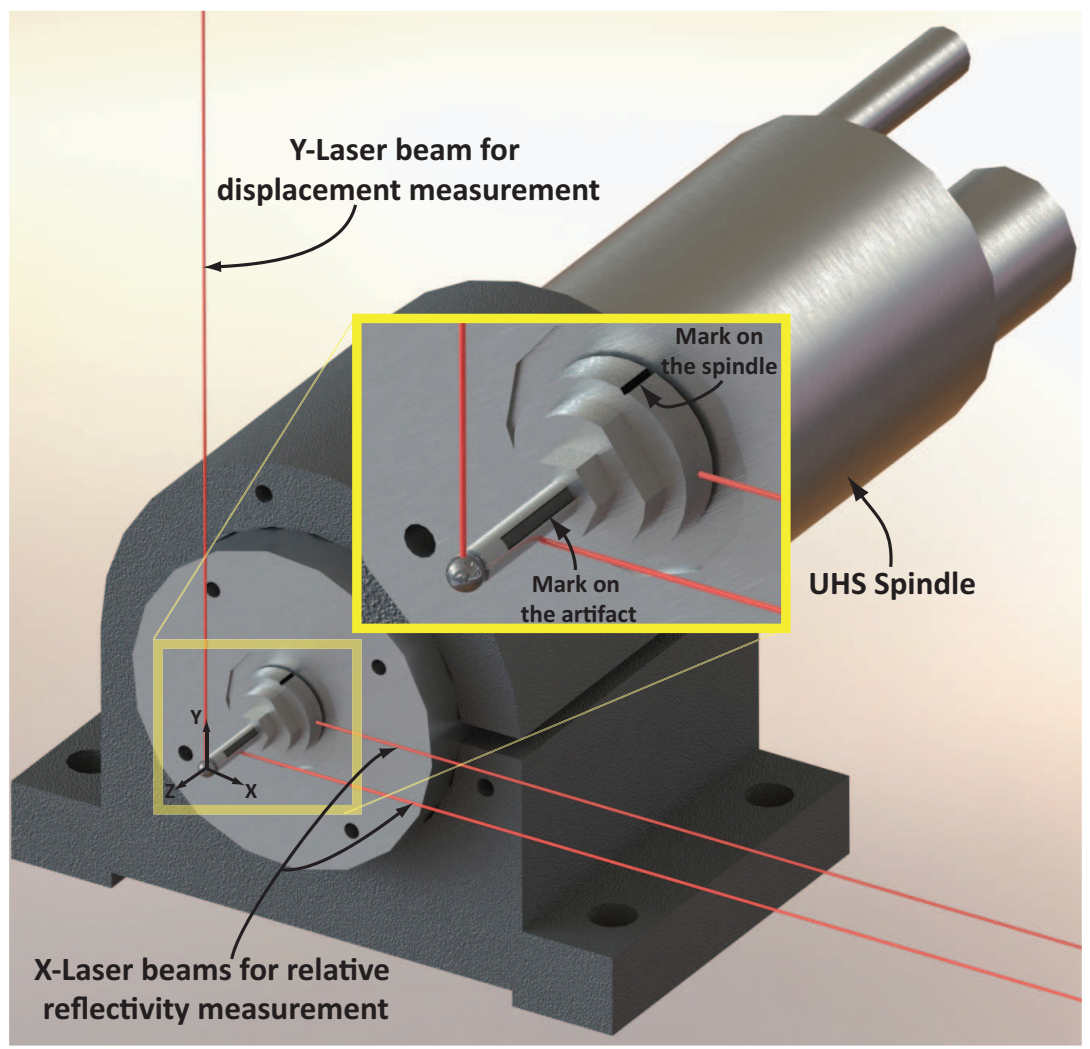

Figure 2: Measurement setup used for implementation of the multi-orientation technique on UHS spindles along a $\operatorname{radial}(Y-)$ direction. In this figure, the marks and laser beams are not shown to scale.

Each time the artifact is removed and re-attached, a high-magnification microscope is used to ensure that the artifact over-hang length is within $\pm 2.5 \mu \mathrm{m}$ across various re-orientations. After attaching the artifact to the spindle, the measurement location of the laser spot on the sphere is fine-tuned using translation adjustments on a kinematic mount to which the laser beam is attached. Adjustments are made until the reflectivity signal of the displacement-measuring LDV is maximized, thus ensuring that the displacement measurement is always conducted perpendicular to the axis average line (see [2]). 


\subsection{Measurement of Relative Angle between Artifact and Spindle}

The measurement of relative angle between the artifact and the spindle is conducted in-situ when the spindle is running at its lowest operational speed. The motivation behind this choice is two folds: First, considering the limited sampling rate of the data acquisition system, the lowest speed provides the highest angular resolution for the re-orientation angle. And second, as observed from dynamic tests conducted on the spindle, this low speed does not correspond to any of the natural frequencies of the spindle, thus minimizing the dynamic response that could cause inaccuracies on the angle determination, and thus, on the separation technique.

The two laser beams are translated along the axial direction such that when the spindle is rotating, each of the physical marks that were made on the stem surface and the rotating spindle surface intersect their respective laser beam paths at some rotational orientation of the spindle. The reflectivity signal is relatively constant, except for the angular locations when either of the marks passes their respective laser beam. At such angular locations, the signal drops sharply, thus producing two distinct notch-shaped features in the reflectivity signal for each revolution.

For a given spindle speed, the shape of the signal drop depends on the physical nature of the mark (width and reflectivity within the mark). Since the two marks are fairly distinct from one another, the resulting signal drops have their own unique pattern. The pattern template corresponding to each of the marks is defined from the data obtained from one of the measurement directions during one of the revolutions. The templates are referred to as the artifact search-pattern and the spindle search-pattern corresponding to the marks on the artifact and the spindle, respectively. A signal processing technique is developed to scan through each successive revolution of the relative reflectivity signal and to identify the exact angular locations within the revolution where the signal shows maximum correlation with each of the search-patterns. The indices corresponding to these locations are stored as spindle- and artifact-markers for that revolution. 
Figure 3 shows a typical reflectivity signal for five revolutions of data, along with the spindle and artifact search-patterns, as defined from the first revolution. The effectiveness of the search algorithm can be seen in the accurate overlap of each of the patterns with their respective spindle and artifact notch features in the other revolutions. The spindle-markers for every revolution corresponding to the matched locations are shown as dotted lines. These spindlemarkers are used to calculate the spindle speed for each revolution. Within a revolution, using the spindle-marker as a reference, the relative angle of the artifact-marker is calculated. These calculations are repeated over all the acquired number of revolutions for that particular orientation. The average value of the calculated relative angles is defined as the relative angle of the artifact with respect to the spindle for that particular orientation. Whenever the orientation is changed, similar relative angle measurements are conducted for the new orientation with the same artifact and spindle search-patterns. The difference between the two relative angles gives the rotation angle $\left(\alpha_{l}\right)$ of the artifact between the re-orientations. This angle is used in the calculations for implementing the multi-orientation method.

\subsubsection{Data Acquisition}

For each orientation, the displacement and reflectivity data are acquired using the National Instruments data acquisition cards NI-6259 (set to $1.25 \mathrm{MHz}$ sampling rate) and NI-6115 (set to $10 \mathrm{MHz}$ sampling rate), respectively. Data acquired from the two cards are synchronized and simultaneously acquired using a LabView ${ }^{\mathrm{TM}}$ code.

\subsubsection{Data Processing}

The data post-processing technique used to separate the fundamental, synchronous and asynchronous components of the radial motion is explained in detail in our previous work [2]. The only difference in this work is that, in order to map data from time-domain to angular-domain (to eliminate the effect of spindle-speed fluctuations), instead of using the pulse-signal from an infra-red 


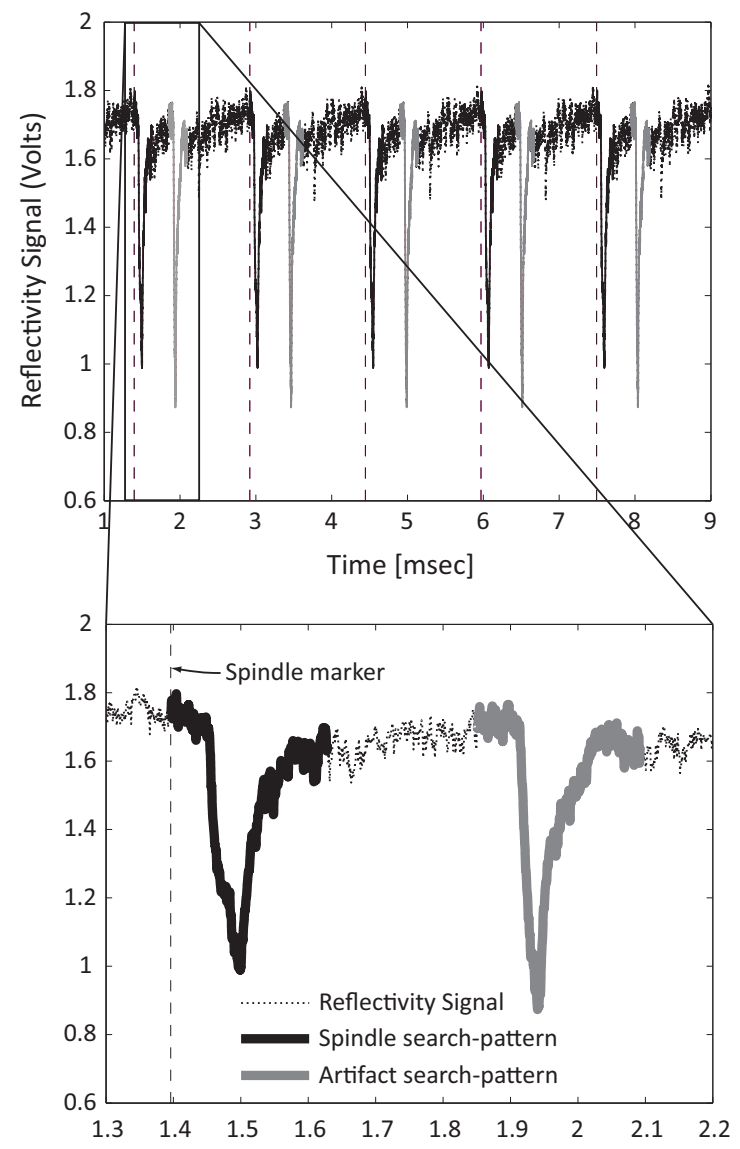

Figure 3: Typical spindle and artifact search-patterns and effectiveness of the search algorithm in pattern-matching.

sensor corresponding to each revolution, the obtained spindle markers are used in this work. The data in the angular-domain is averaged across all the revolutions at each angular location. A sine function is fitted to this data in a least-squares sense and the fundamental component is removed. The remaining data is the synchronous radial motion, which includes both the artifact form error and the synchronous radial spindle error motions. Further post-processing is performed by following the mathematical formulation shown in Section 2 .

As mentioned in Section 2, the multi-orientation method suffers from harmonic suppression at certain harmonics when the denominator approaches zero. 
In theory, for instance when using three re-orientations, the measurement bandwidth arising from the harmonic suppression includes $(k-1)$ harmonics, such that the $k^{\text {th }}$ harmonic satisfies

$$
\left|e^{-j k \alpha_{1}}\right|=1 \quad \text { and } \quad\left|e^{-j k \alpha_{2}}\right|=1
$$

However, in practice, even when it is not zero, a small value of the denominator (in Eq. 9) causes increased errors. Therefore, in order to reduce the uncertainty in the estimation of harmonics, we discarded the harmonics above $k_{l}$, where $k_{l}$ satisfies

$$
\left|e^{-j k_{l} \alpha_{1}}\right|>0.85 \text { and }\left|e^{-j k_{l} \alpha_{2}}\right|>0.85
$$

Hence we considered the practical bandwidth harmonic number $\left(k^{*}\right)$ as the first value of $k_{l}$. Further, since we are interested in measuring a maximum of up to only the first 50 harmonics, a zero phase-shift low pass filter is used to discard all the harmonics greater than 50 throughout the analysis.

\section{Evaluation of the Method}

A successful implementation of the multi-orientation technique (i.e., accurate separation of the artifact form and spindle error motions) requires (1) accurate displacement measurements, keeping all the factors affecting the spindle error motions constant, (2) measurements at the same axial plane of the artifact (i.e., the same circumferential track on the artifact for all re-orientations), and (3) accurate measurement of the artifact rotation angle between the different re-orientations.

The fundamental assumption behind any error-separation technique is that the spindle error motions do not change between the different re-orientations. Sources such as the artifact over-hang length, spindle speed and spindle thermal state can directly contribute to the configuration-to-configuration variations in

spindle error motions. In addition to these factors, in the case of UHS spindles, 
due to the ultra-high speeds, the dynamic effects triggered by the centering errors could also vary the spindle error motions between different configurations. We have addressed most of these issues within the paper in order to evaluate the accuracy of the multi-orientation method.

In this work, we have used a typical UHS spindle to demonstrate the multiorientation technique. This spindle is an electrically-driven hybrid ceramic bearing UHS spindle (Fischer Precise SC1060A) with an operational speed range of $40 \mathrm{krpm}$ to $160 \mathrm{krpm}$. As mentioned earlier, the artifact over-hang length and the circumferential track corresponding to the artifact form error are held within $\pm 2.5 \mu \mathrm{m}$ across various re-orientations. Spindle speed, except for small fluctuations of $\pm 100 \mathrm{rpm}$, is fairly constant and is set using the spindle controller.

Although this spindle is continuously cooled with a coolant and refrigeration unit, even at the steady state, i.e., after the spindle has equilibrated with the ambient conditions, the thermal state of the spindle (as characterized by the inlet/outlet temperatures of the coolant) is known to fluctuate due to the on/off cycling of the coolant temperature controller (see Fig. 4). The range of fluctuation is around $2.5^{\circ} \mathrm{C}$ for the inlet temperature and around $1.8^{\circ} \mathrm{C}$ for the outlet temperature, across the spindle speed range. This thermal cycling could potentially cause fluctuations in the synchronous error motions of the spindle [3]. In order to reduce this effect, the inlet temperature is monitored real-time and the displacement data for each orientation is acquired at multiple (at least ten) thermal states (corresponding to various inlet temperatures) spanning the range of thermal fluctuations, with at least three repetitions for each state. From the displacement data sets, synchronous motion components are separated for each data set using the technique elaborated in [2]. The synchronous motions used for the mathematical formulation shown in Section 2 are the average of the synchronous motions measured at the multiple thermal states, including all the repetitions. Thus, any potential changes in the spindle error motions due to thermal cycling effects are averaged out for each orientation before going through the error separation calculations. Once the artifact form error is calculated from these averaged synchronous motions, the synchronous spindle 


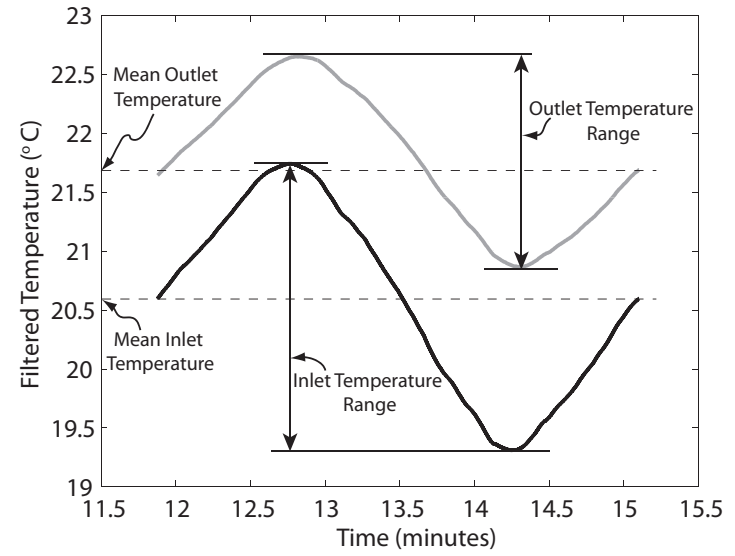

Figure 4: Thermal cycling observed in the tested spindle. Each time instant within this cyclic behavior indicates a thermal state.

error motions for a specific thermal state are calculated by using the original synchronous motions measured for that particular thermal state.

The main factor that could not be controlled for the spindle tested in this paper is the variation in centering errors between different artifact re-orientations. The spindle uses a thread-in style collet, which has been seen to cause relatively large variations in the fundamental amplitude (up to 100\% change) between repeated attachments (see [3]). In addition to contributing to motion at the fundamental frequency, the non-linear dynamic effects due to the centering errors $[3,23,24,26]$ could result in motions at various harmonics of the spindle frequency, i.e., leading to synchronous motions. Hence, variations in centering errors directly affects the accurate measurement of synchronous motions. Hence, as described above, considering the limited sampling rate and possible unwanted dynamic effects due to differences in centering errors across re-orientations, the lowest operational speed of the UHS spindle is used to implement the error separation technique and obtain the artifact form error. This is justifiable since the obtained artifact form remains the same across different speeds and can be directly subtracted from the synchronous motions measured at higher speeds to obtain the spindle error motions.

To evaluate the effectiveness of the multi-orientation technique in error- 
separation for UHS spindles, measurements are conducted on the Fischer Precise spindle at its lowest operational speed of $40 \mathrm{krpm}$ along the fixed-sensitive $Y$ direction for fifteen different (arbitrary) orientations of the artifact. All tests are conducted on the custom-made sphere-on-stem artifact, from both the sphere and stem (cylinder) $(\approx 3 \mathrm{~mm}$ away from the sphere) regions of the artifact for all the fifteen orientations.

The synchronous motion data obtained from the fifteen orientations are used to characterize (1) the effect of the number of orientations (used for separation) on the bandwidth of error separation, and (2) the repeatability of the measured artifact form error. For a given number of orientations, different combinations of measurement angles are selected arbitrarily from the set of fifteen orientations. For a given combination, the corresponding rotation angles are used in Eq.(15) to calculate the bandwidth of error separation.

Those combinations that result in a bandwidth of less than 50 harmonics are considered invalid for further processing. For the valid combinations, the average synchronous motion corresponding to each of the orientations is used within the framework of the mathematical formulation described in Section 2 to obtain the artifact form error. After discarding extreme outliers (using the quartile-based outlier detection method [21]), repeatability of the calculated artifact form error is analyzed for the given number of orientations. Beginning from three-orientation implementation, all possible number of orientations up to fifteen-orientation implementation are studied.

The above analysis is conducted for the data acquired from both the stem and sphere portions of the artifact that are expected to have significantly different form errors. Further, the radial spindle error motions obtained from the two (very different) surfaces of the artifact (separated approximately by 3 mm axial distance) are compared to directly evaluate the effectiveness of the multi-orientation technique in obtaining the spindle error motions. 

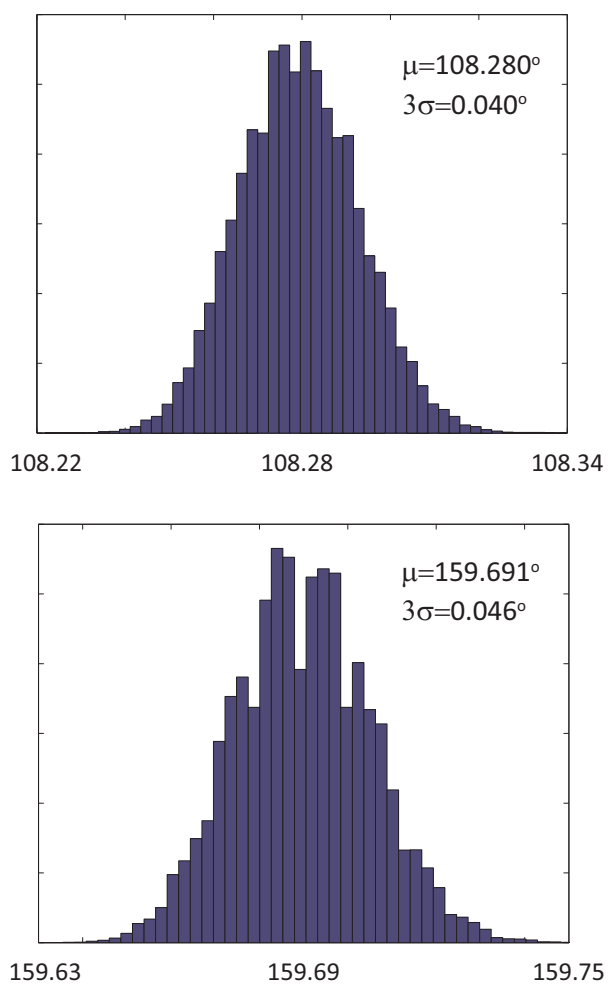

Figure 5: Histograms of the measured relative angles for two of the fifteen re-orientations.

\section{Results and Discussion}

\subsection{Repeatability and Bandwidth}

The relative angle for a given orientation is defined as the average of the relative angles between the artifact- and spindle-markers, measured across all the revolutions for that particular re-orientation. The average includes data from approximately 33,000 revolutions for each re-orientation. Histograms of the relative angles for two of the fifteen re-orientations are shown in Fig. 5. It can be clearly seen and verified using Anderson-Darling normality test that the distribution is of the normal type. This was the case for all re-orientations. Hence, we can use the standard deviation $(\sigma)$ of the relative angle measurements to characterize the variability and angular resolution.

Table 1 shows relative angles for all the orientations, including their $3 \sigma$ 
Table 1: Descriptive statistics of the measured relative angles and the rotation angles for all re-orientations.

\begin{tabular}{cccc}
\hline Orientation & $\begin{array}{c}\text { Relative angle statistics (in degrees) } \\
\mu\end{array}$ & $3 \sigma$ & $\begin{array}{c}\text { Rotation angle (in degrees) } \\
\left.\text { (Total range: }-89^{\circ}-219^{\circ}\right)\end{array}$ \\
\hline 1 & 108.28 & 0.04 & 0 \\
2 & 66.47 & 0.09 & -41.81 \\
3 & 91.12 & 0.07 & -17.16 \\
4 & 19.22 & 0.10 & -89.06 \\
5 & 118.85 & 0.08 & 10.57 \\
6 & 300.62 & 0.15 & 192.34 \\
7 & 236.77 & 0.05 & 128.49 \\
8 & 275.00 & 0.11 & 166.72 \\
9 & 255.08 & 0.09 & 146.80 \\
10 & 270.95 & 0.06 & 162.67 \\
11 & 159.69 & 0.05 & 51.41 \\
12 & 185.16 & 0.06 & 76.88 \\
13 & 219.86 & 0.10 & 111.58 \\
14 & 37.43 & 0.10 & -70.85 \\
15 & 327.08 & 0.13 & 218.81 \\
\hline
\end{tabular}

values. The measured $3 \sigma$ values are less than $0.15^{\circ}$. This indicates that the resolution of the reflectivity-based pattern-detection technique used for the identification of the spindle- and artifact-markers, and hence the resolution of the rotation angles is better than $\pm 0.15^{\circ}$ with $99.7 \%$ confidence. By arbitrarily setting the rotation angle of the first orientation to be zero, the rotation angles of the remaining fourteen re-orientations with respect to the first one are calculated. During these tests, while re-orientating the artifact, specific attention is paid to turn the artifact in such a manner that it covers a wide range of angles ($\left.89^{\circ}-219^{\circ}\right)$. This can be observed from the measured rotation angles. However, other than this, no attempt was made to obtain specific angular orientations.

For a given number of orientations, Figure 6 gives the percentage of the number of combinations whose bandwidth is at least greater than a certain minimum fixed value. The specific percentage values are functions of the angles used in the implementation of the multi-orientation technique. As the number of orientations are increased, a greater percentage of the combinations have bandwidths that lie in a higher harmonic range. For example, from the set of all combinations of 3 orientations for the 15 arbitrary angles used in this 


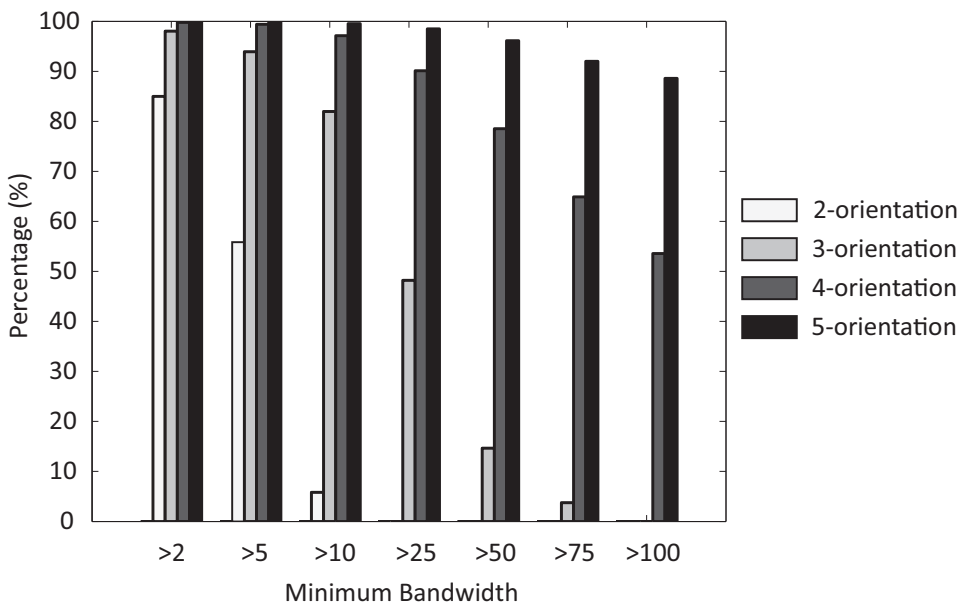

Figure 6: Percentage of combinations for a given number of orientations whose bandwidth is at least greater than a certain minimum fixed value.

study, about $48 \%$ of them have a minimum bandwidth of at least 25 harmonics. However, when using four orientations, about $90 \%$ of the combinations have a minimum bandwidth of at least 25 harmonics. Further, when the number of orientations is increased to five, close to $97 \%$ of the five-orientation combinations have bandwidth greater than 50 harmonics.

As mentioned earlier, for further processing, to ensure that the first 50 harmonics are captured as accurately as possible, we have considered only those combinations with a bandwidth greater than 50 harmonics as being valid (note that this can be assessed a priori from the determined orientation angles). Figure 7 shows the repeatability of the artifact form errors for both the sphere and stem portions as obtained from valid combinations of five orientations after rejecting the outliers. The range of variation of the artifact form error at each angular index is recorded and its average value across the entire rotation is used as the metric for repeatability. Figure 8 shows the histograms of the range of variation of the artifact form errors on both the sphere and stem portions of the artifact across the entire circumference for the five-orientation implementation of the method. Using the Anderson-Darling test for normality, both the distributions are confirmed to be of the normal type, with their standard deviations 

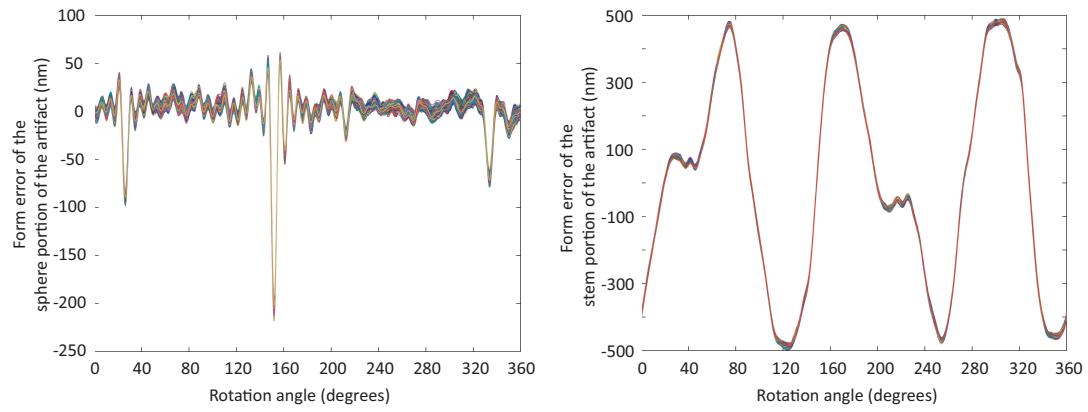

Figure 7: Repeatability of the measurement of artifact form errors for the five-orientation implementation: (a) Measurement from the sphere, (b) Measurement from the stem.

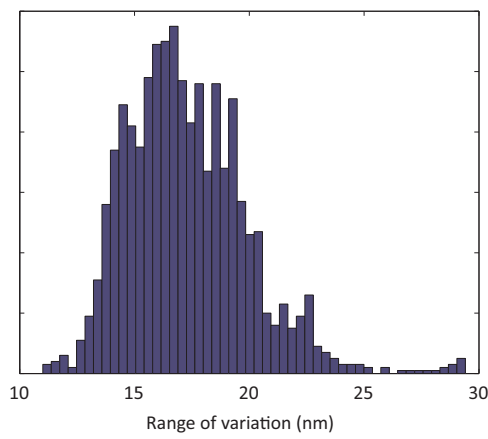

(a)

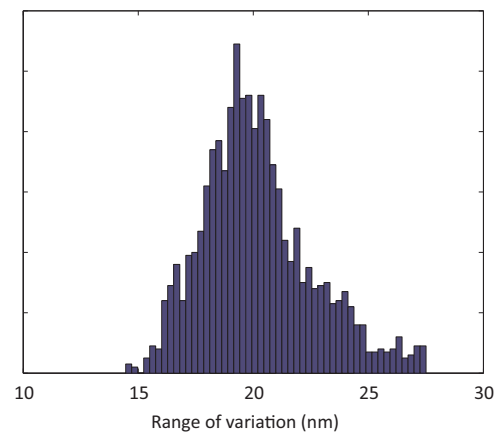

(b)

Figure 8: Histograms of the range of variation of the artifact form errors across the circumference for the five-orientation implementation: (a) Measurement from the sphere, (b) Measurement from the stem.

being $2.3 \mathrm{~nm}$ and $2 \mathrm{~nm}$, respectively. The average value is about $17 \mathrm{~nm}$ for the sphere portion and $20 \mathrm{~nm}$ for the stem portion. It is worth noting that these values are similar even though the artifact form errors are significantly different in both shape and magnitude for the sphere and stem portions.

There could be a few reasons for the observed variability. As evaluated in $[1$, 2], any noise in the measurement of the synchronous motions due to environment effects, laser beam alignment and curvature effects leads to uncertainty in the synchronous motion data. From [2], the combined standard uncertainty for a single measurement of the synchronous radial motion due to the environmental effects, laser beam alignment and curvature effects is $3.6 \mathrm{~nm}$. Using a coverage factor of 2 , this corresponds to an expanded uncertainty of $\pm 7.2 \mathrm{~nm}$ and hence 
a range of variation of $14.4 \mathrm{~nm}$. In the above example, for each measurement of the artifact form error, five measurements of the synchronous radial motion are used. Hence the range of variation in the measured artifact form error due to environment effects, laser beam alignment and curvature effects is $14.4 / \sqrt{5}=$ $6.4 \mathrm{~nm}$. In addition to these sources, there are two other sources that could add to the variability. First, the artifact form error could be measured from slightly different circumferential tracks due to small differences (within $\pm 2.5 \mu \mathrm{m}$ ) in the axial measurement locations for the various re-orientations. Second, even though the lowest operational speed of the spindle was used, the fundamental component of the measured motions (caused by the centering errors and the resulting dynamic effects) between re-orientations varied as much as $100 \%$ [3]. These variations in centering errors and their dynamic effects [3, 23, 24, 26] could result in differences in the synchronous motions between re-orientations, and hence, cause variability in the measured artifact form error when different sets of re-orientations are used.

In Fig. 9, the average value of the range of variation is plotted with respect to the number of orientations. As the number of orientations increases, the average value reduces significantly. This could be explained by the fact that when greater number of orientations are used, the formulation of the combined sum (Eq. 13) naturally causes an averaging effect, thus reducing the uncertainty (caused by various un-biased noise sources in each orientation) in the combined sum (see [22]). In the case of lower number of orientations, variations between orientations would not be completely averaged out, thus causing increased variability between the artifact form errors obtained from different combinations. It was further observed that when the number of orientations is increased, the average artifact form error (calculated from all valid combinations of a given number of orientations) seems to converge to the artifact form error obtained from combining all the 15-orientation measurements. Therefore, if higher measurement accuracy is desired for both the artifact form and spindle errors, a larger number of orientations should be considered. For instance, if 12 orientations are used, the average variation reduces to below $5 \mathrm{~nm}$. 


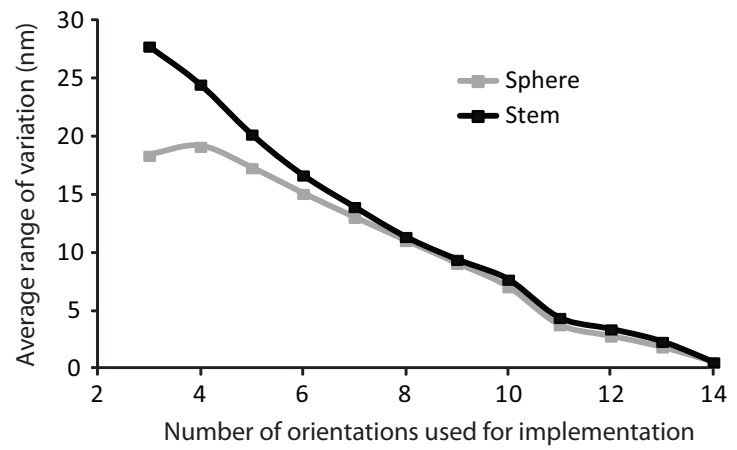

Figure 9: Average value of the range of variation of the artifact form errors (across valid combinations) for the different number of orientations.

For ease of practical implementation of the multi-orientation technique, we desire to choose the minimum number of re-orientations that allows calculation of the artifact form error within a specified average range of variation (e.g., 20 $\mathrm{nm})$. From Fig. 9, the desired average range repeatability level is attained if a five-orientation implementation is used. Hence, for further analysis within this section, the artifact form error is obtained as an average from different combinations of the five-orientation implementation.

\subsection{Spindle error motions from the sphere and stem portions of the artifact}

To demonstrate the effectiveness of the multi-orientation technique in calculating the spindle error motions, the artifact form errors for the sphere and stem portions are subtracted from their respective synchronous motions measured at various thermal states for a certain orientation. The synchronous motions and the calculated spindle error motions (after removing the form error) as measured from the sphere and the stem are shown for ten different thermal states (with three repetitions per state) in Fig. 10. Although the artifact form errors on the sphere and stem portions are significantly different (up to ten times), it can be observed that the characteristic features of both the form errors are completely removed from their respective synchronous motions, thus resulting in spindle error motions that visibly have the same characteristic features and magnitude range. 
To further quantify the effectiveness of the technique, Fig. 11 shows the average spindle error motions (averaged across the different thermal states) as calculated from the sphere and stem portions. The mean difference in magnitude between the two spindle error motion profiles across the rotation angles is calculated to be $2.5 \mathrm{~nm}$. Also, it can be observed that at those angular locations where differences are observed, the error motions calculated from the sphere portion are invariably higher than those calculated from the stem portion. These slight differences could be due to (a) the synchronous tilt error motions of the spindle, and (b) the non-linear dynamics effects due to centering errors, since the measurement location on the sphere is axially further out by approximately $3 \mathrm{~mm}$ compared to the stem location. 

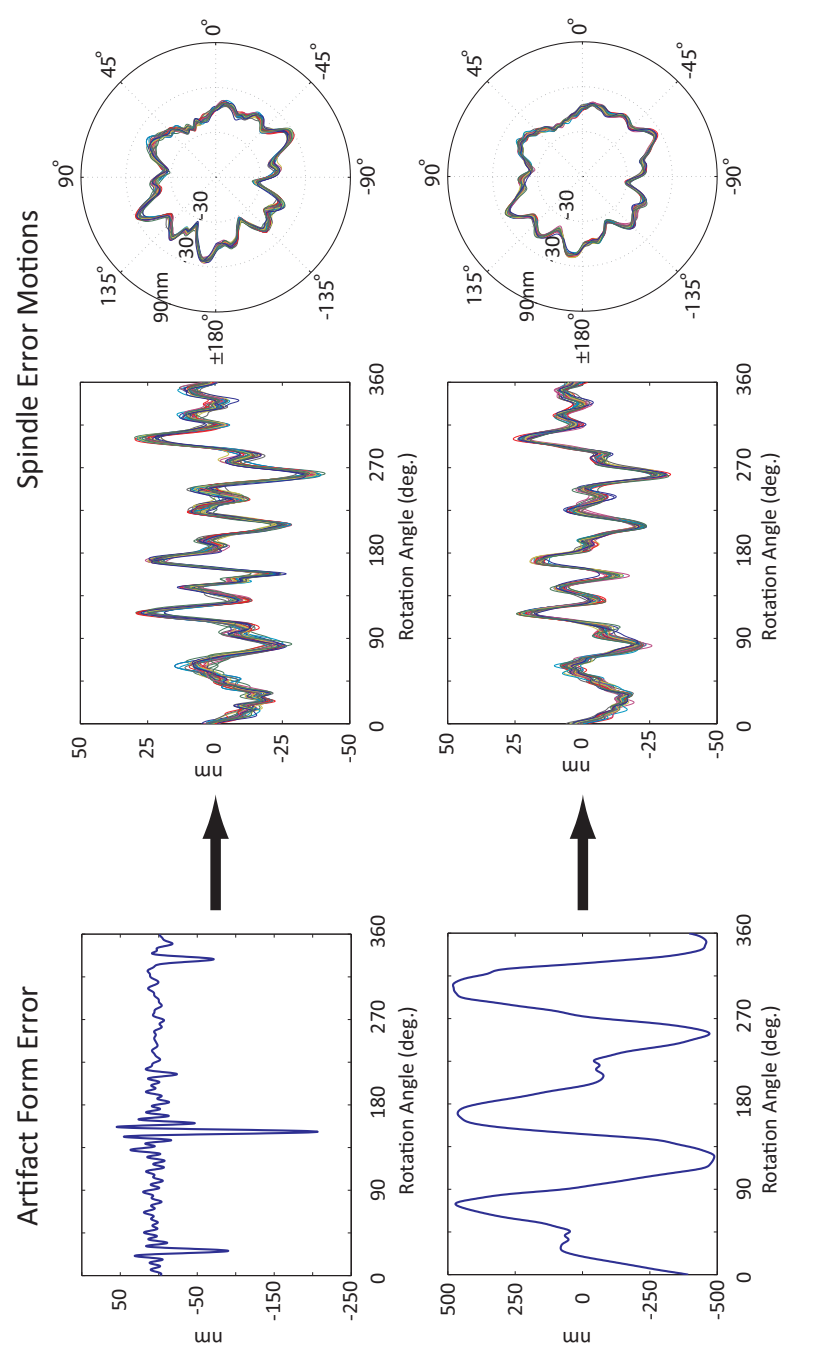

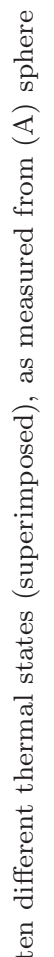

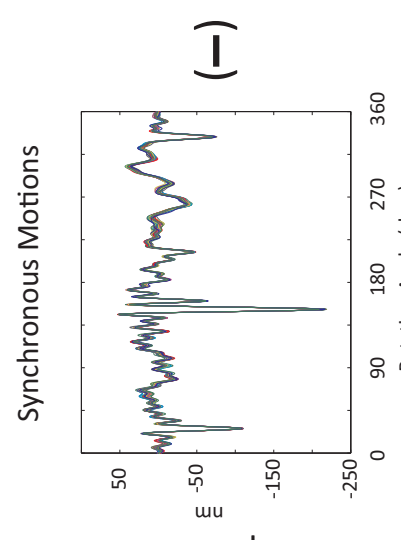

әләчds

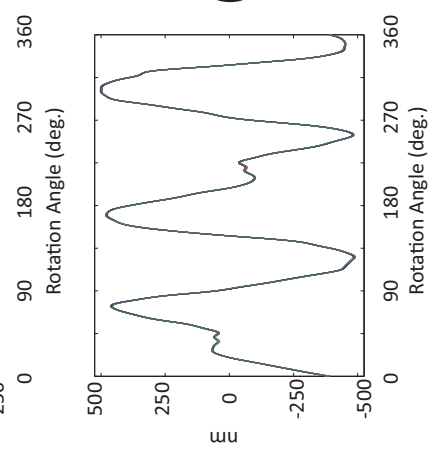

แวเร

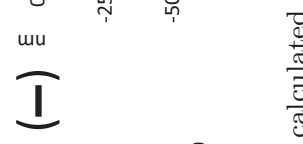

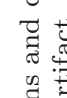

范

है

ติ 0

ํํㄹ

穿茪

की है

웅

๑ิ

的望䒕 


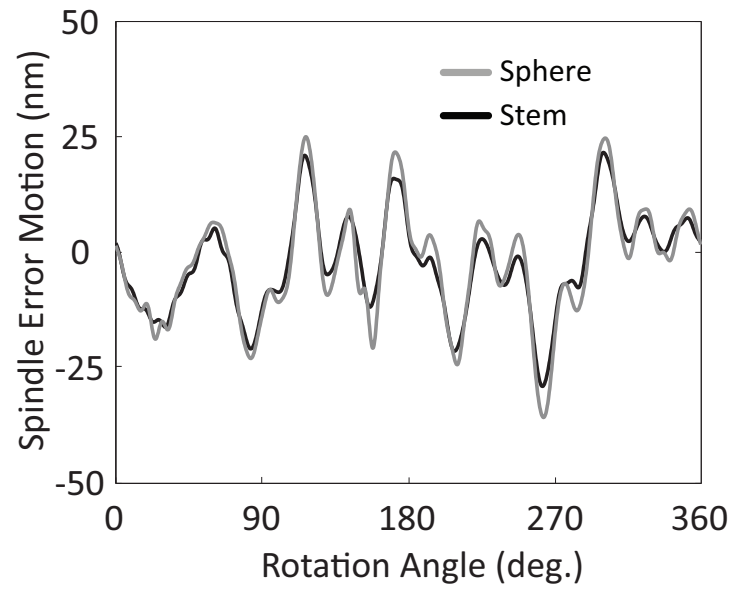

Figure 11: Spindle error motion calculated from the sphere and stem portions of the artifact.

\section{Case Study: Error Motions of an Ultra-High Speed Spindle at Operational Speeds}

The previous section demonstrated the effectiveness of the artifact form error removal at the lowest operating speed of the spindle. The procedure for obtaining the error motions at ultra-high speeds is similar to what was explained in the previous section. The multi-orientation method is implemented with a certain number of orientations and the average artifact form error with the desired number of harmonics is obtained for the lowest operational speed. For any other spindle speed, this form error is used as a template and interpolated to the angles at which synchronous motion is measured for the higher speed. Spindle error motions are then calculated by subtracting the artifact form from the synchronous motion data.

To demonstrate this procedure, spindle error motions are determined along two orthogonal fixed-sensitive radial directions $(X$ and $Y)$ at four spindle speeds (40 krpm, $90 \mathrm{krpm}, 120 \mathrm{krpm}$ and $150 \mathrm{krpm}$ ). Because we have only two LDV systems, the $X$ - and $Y$-measurements have to be conducted separately. The multi-orientation error separation procedure is first completed along one of the directions (say $Y$-direction). Then, the same procedure, however with a different 
set of (arbitrary) orientations, is repeated along the $X$-direction.

Prior to conducting any measurements, $X$ - and $Y$-laser beams have to be aligned perpendicular to each other. The $X$ - and $Y$-laser beams are from two independent laser sources, each with differential fiber-optic carriers. For singlepoint measurements, one of the fiber-optic carriers is blocked with a mirror.

Assuming that the $Y$-direction measurements were conducted first, the setup shown in Fig. 2 was used as the starting point for alignment. The $Y$-laser beam not used for the measurement was capped off with a mirror. The $X$-laser beam that will be later used for displacement measurement was kept open, while the other $X$-laser beam was blocked off with a pentaprism and mirror arrangement (as explained in [2]). The measurement $X$-laser beam was translated using a precision translation stage to the sphere portion of the artifact. Using the 6axis kinematic mounts on which the laser beams were mounted, angular and translational adjustments were made such that both the measurement $X$ - and $Y$-laser beams were perpendicular to two of the surfaces of a three-faced corner cube retroreflector ${ }^{4}$, while simultaneously being perpendicular to the average surface of rotation (i.e., axis average line). This procedure was iterative and was explained in [2].

Once the alignment procedure was completed, the measurement lasers along $X$ and $Y$ become perpendicular to each other and with respect to the axis average line. The pentaprism and mirror arrangement that blocked the second $X$-laser beam was then removed. Subsequently, the two $X$-laser beams (mounted on precision translation stages) were translated such that one of them is focused on the stem portion of the artifact that has the physical mark, while the other is focused on the rotating surface of the spindle that has the physical mark. Single-point $Y$-displacement and relative $X$-reflectivity were then simultaneously measured as part of the $Y$-axis measurement set.

Once all the $Y$-measurements were completed, without disturbing the measurement $Y$-laser beam, the $X$-laser was shifted from the relative mode to the

\footnotetext{
${ }^{4}$ The faces of the corner cube are orthogonal to each other within an angle of 10 seconds
} 
single-point mode by capping off the second $X$-laser beam with a mirror. The measurement $X$-laser beam was moved over to the sphere portion of the artifact and was focused on the sphere. Since the measurement $Y$-laser beam remained untouched throughout this process, it was used as a reference. The corner-cube was used again and one its faces was made perpendicular to the measurement $Y$-laser beam. The alignment of the measurement $X$-laser beam was then fine-tuned to make it perpendicular to the adjacent face of the cornercube, and thus, to the $Y$-direction measurement. The $Y$-laser was then split into two beams by removing the mirror-cap from the capped-off fiber. The first $Y$-laser beam, mounted on a precision translation stage, was first translated and focused on the stem portion of the artifact with the black mark. The other $Y$ laser beam was attached to another precision translation stage mounted on the frame, and was translated and focused on the rotating surface of the spindle that has the physical mark. The setup for the $X$-measurements is shown in Fig. 12 . Single-point $X$-displacement and relative $Y$-reflectivity are the simultaneously measured as part of the $X$-axis measurements.

By going through this procedure, all the measurements were first completed along the $Y$-direction, followed by the measurements along the $X$-direction. Spindle error motions along the rotating-sensitive direction were calculated by projecting the measured motions along $X$ - and $Y$-directions onto a rotating vector $[19,20]$.

Five different orientations are measured for each direction. After the measurements from all orientations are completed, using the calculated rotation angles, it is checked to ensure that the bandwidth covers at least the $50^{\text {th }}$ harmonic. If needed, additional measurements are conducted from more orientations of the artifact, until a valid combination of at least five orientations could be obtained without harmonic losses below 50 harmonics. The artifact form error is then calculated from the measurements conducted at $40 \mathrm{krpm}$ using the valid combinations of five orientations. This form error is used as a template, and it (interpolated version) is used to remove the form error from the synchronous motions measured at the four tested higher speeds. The ef- 


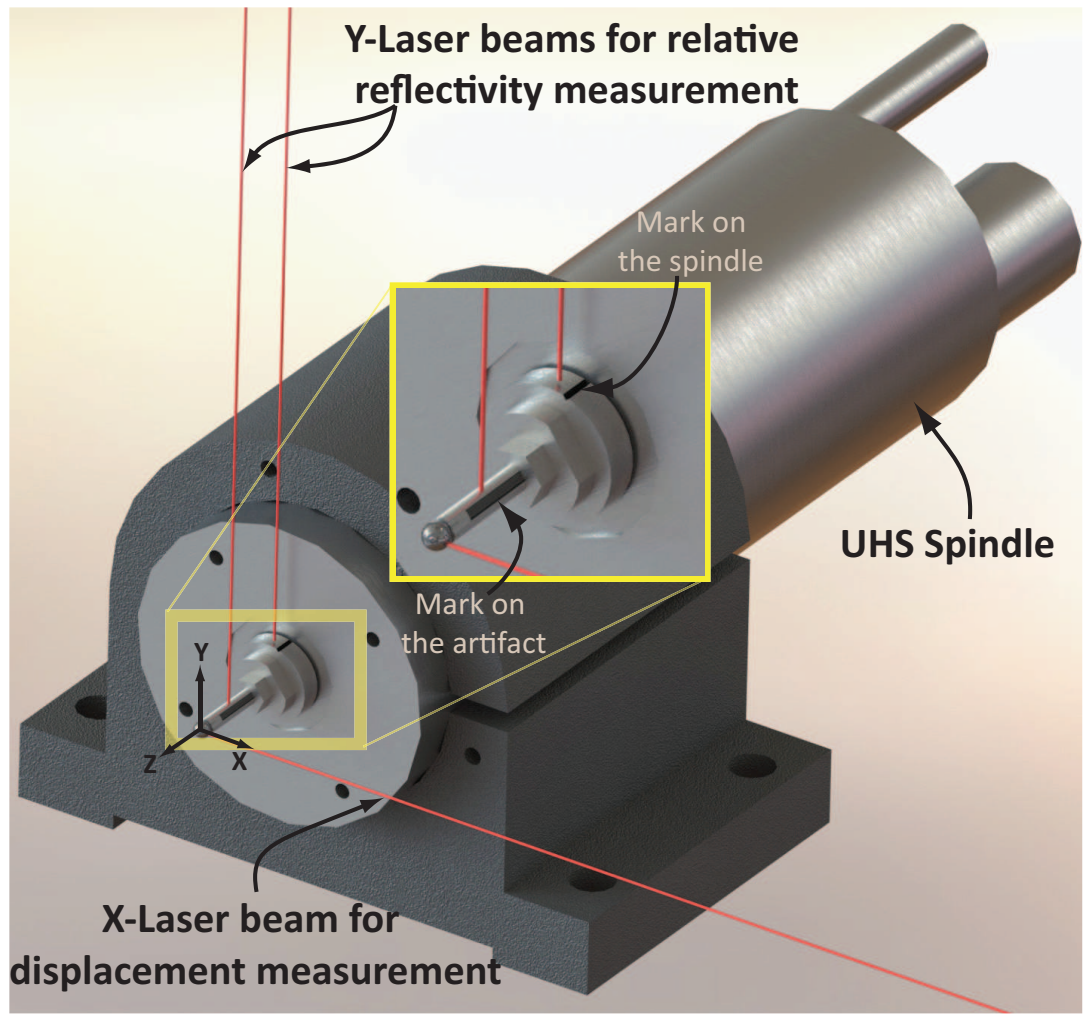

Figure 12: Measurement setup for implementation of the multi-orientation technique on UHS spindles along radial $X$-direction.

fectiveness of this procedure is demonstrated in Fig. 13 at $90 \mathrm{krpm}, 120 \mathrm{krpm}$ and $150 \mathrm{krpm}$ for measurements conducted along the $Y$-direction. Both the synchronous motions as well as the spindle error motions (after form error removal) are shown for a given thermal state. It can be clearly seen that all the features of the artifact form (such as the notches) are completely removed from the raw measurements. The spindle error motions along the $X$ and $Y$ fixed-sensitive directions, and along the rotating-sensitive direction, are shown in Fig. 14 for all the four speeds. The speed-dependent nature of both the shapes and the magnitudes of these error motions is quite evident from the plots. 

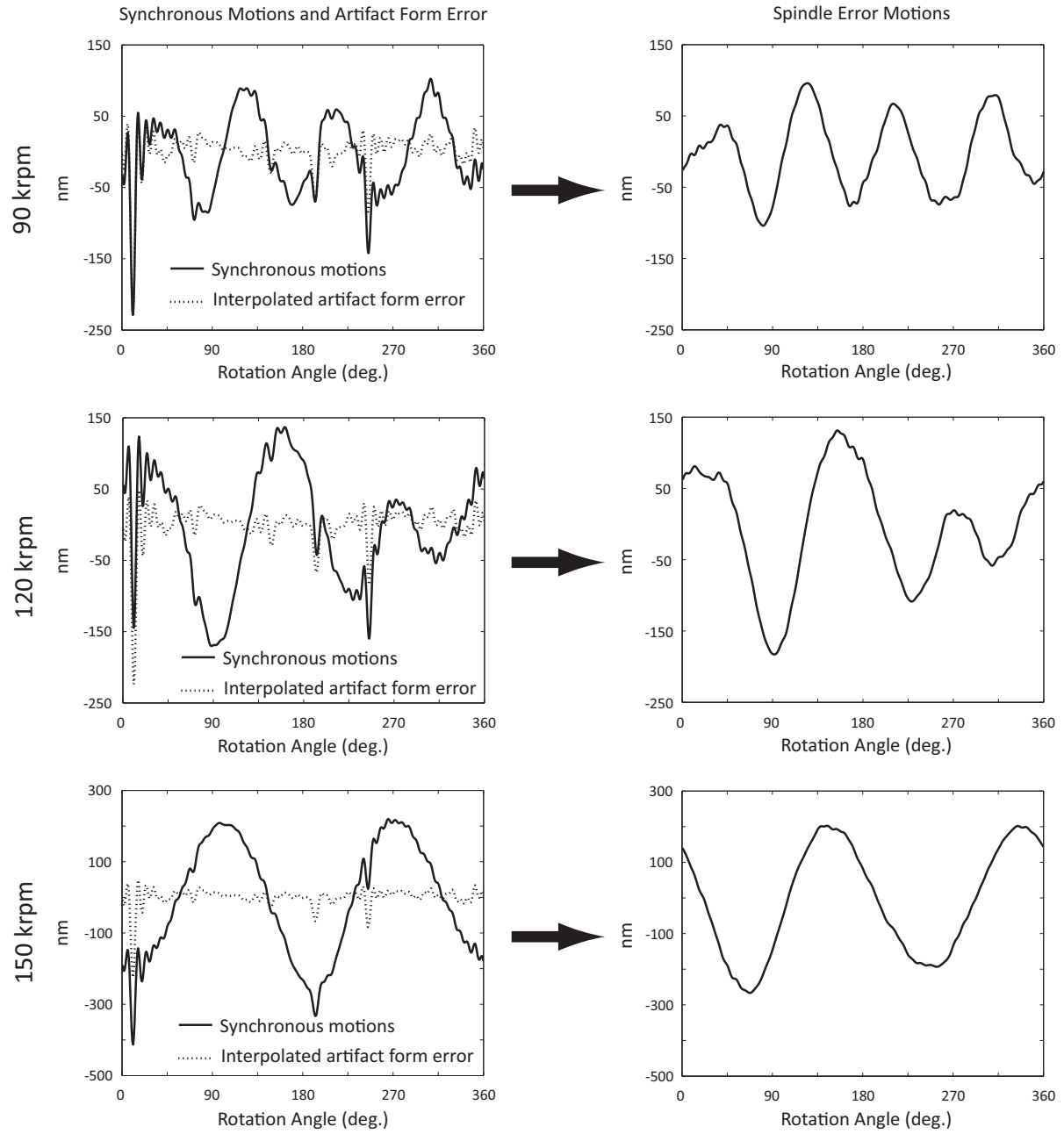

Figure 13: Synchronous motions and calculated spindle error motions along radial $Y$-direction for $90 \mathrm{krpm}, 120 \mathrm{krpm}$ and $150 \mathrm{krpm}$.

\subsection{Time required for measurements}

The total time period required to complete a set of measurements depends on various factors: (a) thermal cycling period of the spindle and the time taken for the spindle to reach thermal equilibrium at the required speed, (b) number of orientations required, (c) measurement hardware: delay in acquisition/saving, and (d) number of Laser Doppler Vibrometers that are available for use.

For the spindle tested in this paper, the thermal cycling period is approx- 

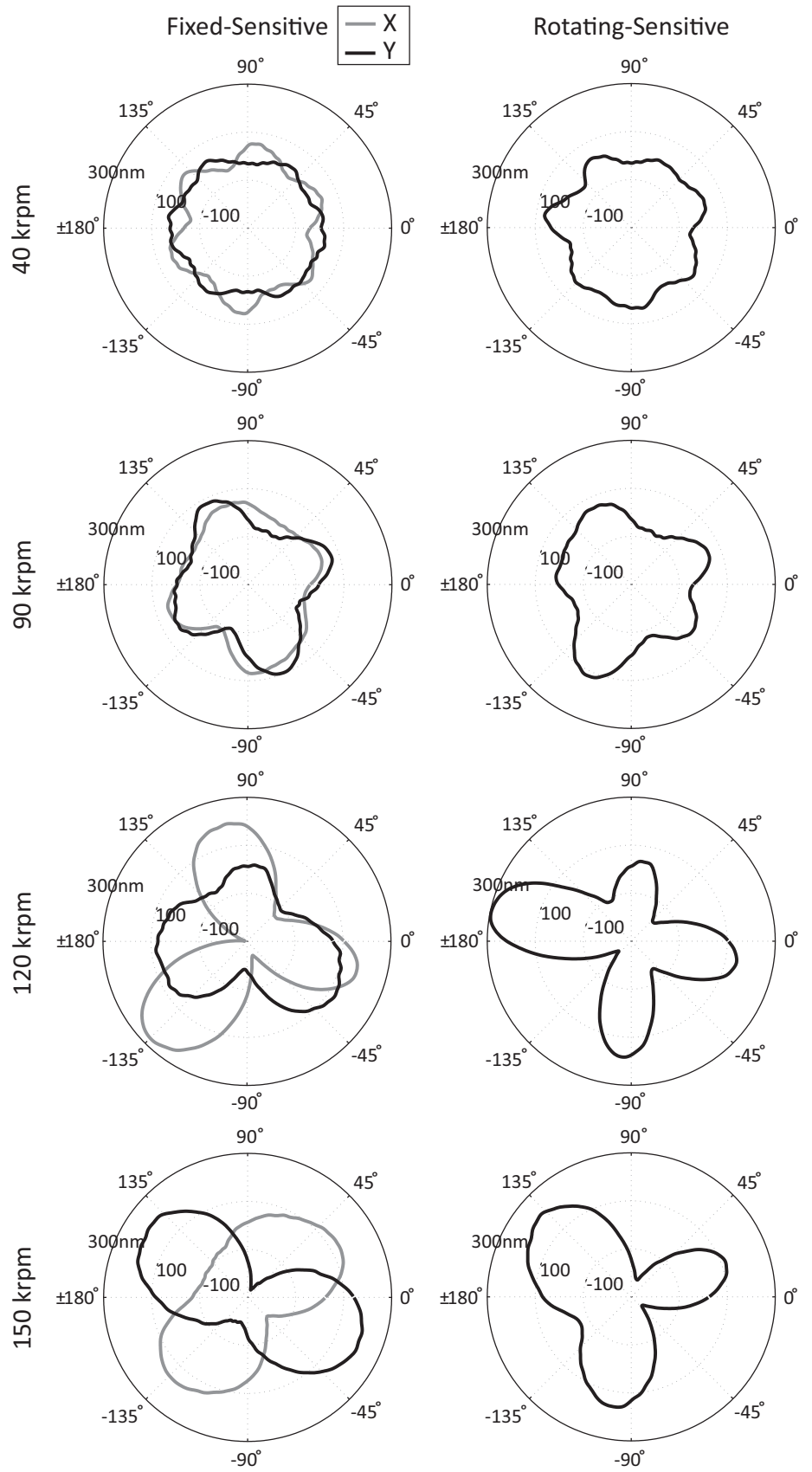

Figure 14: Spindle error motions along fixed-sensitive directions $(X$ and $Y$ ) as well as along the rotating-sensitive direction for all the tested speeds. 
imately 3 minutes. Using the instrumentation hardware that is used, it takes about 15 minutes to acquire three repeats of data for ten thermal states. Changing between orientations, fine-tuning the LDV orientation and waiting for thermal equilibrium takes about 45 minutes. Since there were only two LDVs available, for each setup, measurements were conducted for only one axis: one of the LDVs was used to measure displacement along the axis, while the second one was used for measurement of angular reference. Overall, on average it takes a little over an hour to complete the measurement for one orientation along one axis. Multi-sensor separation techniques could potentially take significantly less time. However, to conduct measurements equivalent to a five-orientation technique would require at least five sensors. The cost of having such a set up with multiple sensors (especially with pico-meter level resolution) would be prohibitively expensive.

\section{Summary and Conclusions}

This paper presented an arbitrary-angle multi-orientation error separation technique (AA-MOES) to remove the artifact form error from the radial spindle measurements to obtain the radial spindle error motions. The presented novel implementation approach relies on in-situ measurement of the arbitrarily-set angular orientation of the artifact with respect to the spindle, and hence, does not require any special precision fixtures for setting specific angles. The applicability of the technique was demonstrated by measuring synchronous error motions of a miniature UHS spindle. A thorough experimental evaluation of the presented technique is conducted and its effectiveness at measuring error motions when the spindle is operating at ultra-high speeds was characterized.

Overall, it was concluded that the presented AA-MOES technique is capable of isolating spindle error motions from artifact form errors accurately, including at ultra-high operating speeds of the miniature spindles.

The following specific conclusions can be drawn from the work presented in this paper. 
- Even though the artifact form errors on the sphere and stem portions are significantly different (up to ten times), the spindle error motions obtained from both locations (which are axially offset by $\approx 3 \mathrm{~mm}$ ) are similar in shape and within $5 \mathrm{~nm}$ in magnitude, thus demonstrating the effectiveness of the technique.

- The harmonic range (bandwidth) of the separation technique increases with the number of tested orientations. For the given set of angles tested in this work, close to $97 \%$ of the combinations of the five-orientation implementation have bandwidths greater than 50 harmonics.

- The average range of variation of the estimated artifact form error across the various combinations for a given number of orientations reduces significantly as the number of orientations increases. It changes from 20 $\mathrm{nm}(17 \mathrm{~nm})$ on the stem (sphere) portion for the five-orientation implementation to below $5 \mathrm{~nm}$ on both the stem and sphere portions for the eleven-orientation implementation. For the given set of angles tested in this work, if a certain level of repeatability is desired for the artifact form error, one can choose the minimum number of orientations required to attain that level.

- At small number of orientations, the stem portion of the artifact (that has a larger form error compared to the sphere portion) results in larger average range of variation. But once the number of re-orientations increases beyond eight, the average range of variations become similar.

- As a principle, considering both the angular resolution of orientation angle measurements and accuracy of the form error determination, a low spindle speed within the operating range of the particular spindle tested should be chosen. Furthermore, it is preferable to conduct dynamic tests on the spindle to ensure that the chosen speed does not coincide with any of the resonances of the spindle assembly to subside the negative impact of the spindle dynamics on measurement accuracy. The form error determined 
at that low speed can then be used at any operational speed for isolating spindle error motions. In this work, by using the form error at $40 \mathrm{krpm}$ as a template, spindle error motions at ultra-high speeds were successfully quantified along both the fixed-sensitive $(X, Y)$ and rotating-sensitive directions at $90 \mathrm{krpm}, 120 \mathrm{krpm}$ and $150 \mathrm{krpm}$.

- With this approach, since the artifact form error can be successfully removed if a certain minimum number of orientations are used, less precise (lower cost) artifacts can be utilized for the spindle error motion measurements. Indeed, the shank portion of the micro-tools themselves would be sufficient for providing very relevant data directly without using a specific precision artifact.

- Further, the multi-orientation error separation technique presented here can be directly applied for measuring spindle error motions of macro-scale spindles without any modifications, thus providing an elegant approach that does not require high precision fixtures and precise setting of angles.

\section{Acknowledgments}

The authors wish to thank Mr. Sudhanshu Nahata, Ph.D. candidate, Carnegie Mellon University, PA, for his help during experimentation and his insightful comments. The authors would like to acknowledge the assistance of Prof. Bulent Arda Gozen of Washington State University for his assistance during data collection. This work was funded in part by National Institute of Standards and Technologies (NIST) award \#70NANB12H208 (Ozdoganlar).

\section{References}

\section{References}

[1] K. P. Anandan, A. Tulsian, A. Donmez, O. B. Ozdoganlar, A technique for measuring radial error motions of ultra-high-speed miniature spindles used for micromachining, Precision Engineering 36 (1) (2012) 104-120. 
[2] K. P. Anandan, O. B. Ozdoganlar, A methodology for measuring axial and radial error motions when using miniature ultra-high-speed (UHS) micromachining spindles, Precision Engineering 37 (1) (2013) 172-186.

[3] K. P. Anandan, O. B. Ozdoganlar, Analysis of error motions of ultra-highspeed (UHS) micromachining spindles, International Journal of Machine Tools and Manufacture 70 (2013) 1-14.

[4] C. J. Evans, R. J. Hocken, W. T. Estler, Self-Calibration: Reversal, Redundancy, Error Separation, and Absolute Testing, CIRP AnnalsManufacturing Technology 45 (2) (1996) 617-634.

[5] R. R. Donaldson, A simple method for separating spindle error from test ball roundness error, Annals of CIRP 21 (1972) 125-6.

[6] D. Whitehouse, Some theoretical aspects of error separation techniques in surface metrology, Journal of Physics E (Scientific Instruments) 9 (7) (1976) 531-6.

[7] J. G. Salsbury, Implementation of the Estler face motion reversal technique, Precision Engineering 27 (2) (2003) 189-194.

[8] E. R. Marsh, D. A. Arneson, D. L. Martin, A comparison of reversal and multiprobe error separation, Precision Engineering 34 (1) (2010) 85-91.

[9] E. R. Marsh, J. Couey, R. R. Vallance, Nanometer-level comparison of three spindle error motion separation techniques, Journal of Manufacturing Science and Engineering, Transactions of the ASME 128 (1) (2006) 180187.

[10] M. J. Jansen, P. H. J. Schellekens, B. De Veer, Advanced spindle runoutroundness separation method, Series on Advances in Mathematics for Applied Sciences, 57 (2001) 212-219.

[11] G. X. Zhang, R. K. Wang, Four-point method of roundness and spindle error measurements, CIRP Annals-Manufacturing Technology, 42 (1) (1993) 593-596. 
[12] G. X. Zhang, Y. H. Zhang, S. M. Yang, Z. Li, A multipoint method for spindle error motion measurement, CIRP Annals-Manufacturing Technology, 46 (1) (1997) 441-445.

[13] C. Linxiang, W. Hong, L. Xiongua, S. Qinghong, Full-harmonic error separation technique, Measurement Science and Technology, 3 (12) (1992) 11291132.

[14] C. Linxiang, The measuring accuracy of the multistep method in the error separation technique, Journal of Physics E: Scientific Instruments, 22 (11) (1989) 903-906.

[15] S. Tong, Two-step method without harmonics suppression in error separation, Measurement Science and Technology, 7 (1996) 1563-1568.

[16] W. Gao, S. Kiyono, T. Sugawara, High-accuracy roundness measurement by a new error separation method, Precision Engineering, 21 (2-3) (1997) $123-133$.

[17] W. Zhao, J. Tan, Z. Xue, S. Fu, SEST: A new error separation technique for ultra-high precision roundness measurement, Measurement Science and Technology, 16 (3) (2005) 833-841.

[18] R. Grejda, E. Marsh, R. Vallance, Techniques for calibrating spindles with nanometer error motion, Precision Engineering 29 (1) (2005) 113-123.

[19] ASME B89.3.4-2010 Axes of Rotation: Methods for Specifying and Testing (2010).

[20] ISO 230-7:2006 Test code for machine tools Part 7: Geometric accuracy of axes of rotation (2006).

[21] NIST/SEMATECH e-Handbook of Statistical Methods, http://www.itl.nist.gov/div898/handbook/, 2012.

[22] W. T. Estler, C. Evans, L. Shao, Uncertainty estimation for multiposition form error metrology, Precision Engineering 21 (2) (1997) 72-82. 
[23] B. Bediz, B. A. Gozen, E. Korkmaz, O. B. Ozdoganlar, Dynamics of ultrahigh-speed (UHS) spindles used for micromachining, International Journal of Machine Tools and Manufacture, 87 (2014) 27-38.

[24] B. Knapp, D. Arneson, Dynamic Characterization of a Micro-machining Spindle, International Conference on Micromanufacturing (ICOMM) 2014, No. 108.

[25] S. Cappa, D. Reynaerts, F. Al-Bender, A sub-nanometre spindle error motion separation technique, Precision Engineering, 38 (3) (2014) 458-471.

[26] Z. Yang, J. Hong, J. Zhang, M. Y. Wang, Research on the rotational accuracy measurement of an aerostatic spindle in a rolling bearing performance analysis instrument, International Journal of Precision Engineering and Manufacturing, 15 (7) (2014) 1293-1302. 